\title{
Bodengeographische Beobachtungen zur pleistozänen und holozänen Vergletscherung des Westlichen Tienshan (Usbekistan)
}

\author{
Wolfgang Zech, Rupert Bäumler, Oksana Savoskul, Anatoli Ni \& Maxim Petrov*) \\ Pleistocene, Holocene, Glaciation, Tienshan, Uzbekistan
}

\begin{abstract}
Kurzfassung: Im Oigaing-Tal zwischen Ugamsky- und Pskemsky-Gebirge nordöstlich von Taschkent (West-Tienshan, Usbekistan) wurden bodengeographische Untersuchungen zur pleistozänen und holozänen Vergletscherung durchgeführt. Eindeutige Endmoränen der letzten Hauptvergletscherung konnten im Bereich des Zusammenflusses von Maidan und Oigaing in 1500 - $1600 \mathrm{~m}$ ü. M. nachgewiesen werden mit mächtigen, bis in $80 \mathrm{~cm}$ Tiefe tiefgründig verwitterten Bodenbildungen. Vergleichbare Ablagerungen vermutlich hochglazialer bzw. spätglazialer Genese finden sich auch talaufwärts im Mündungsbereich zahlreicher Seitentäler (Beschtor-, Tekesch-, Aütor-Tal) in das Oigaing-Haupttal. Die Seitentäler weisen in 2500 bis 2700 m spätglaziale Stirn- und Grundmoränen auf. Die Böden dieser Ablagerungen sind ebenfalls bis in $40-60 \mathrm{~cm}$ Tiefe stark verwittert und verbraunt. Den rezenten Gletschern, die bis auf ca. 3000 - $3200 \mathrm{~m}$ herabreichen, sind weitere Moränen holozänen bzw. neuzeitlichen Ursprungs vorgelagert mit flachgründigen, z. T. initialen Bodenbildungen, die vermutlich mit Gletschervorstößen während der sogenannten „Kleinen Eiszeit" mit einem Maximum in den Alpen um 1850 und im mittleren Holozän um 2000 bzw. 4000 a BP übereinstimmen. Im unteren Seitental des Barkrak sind oberhalb von hochglazialen Eisrandlagen (>2850 m) interglaziale, sehr stark verwitterte und rubefizierte Bodenbildungen aus altquartären Schottern erhalten, die von einer spätpleistozänen Solifluktionsdecke überfahren wurden. Der obere Talverlauf ist dagegen oberhalb dieser hochglazialen Eisrandleisten durch mächtige Geschiebe gekennzeichnet. Sie sind Zeugen älterer, im Vergleich zur jüngsten Hauptvergletscherung wesentlich mächtigerer Vereisungen. Die dazugehörigen Moränen konnten jedoch nicht gefunden werden.
\end{abstract}

\section{[Soil geographic studies for the Pleistocene and Holocene glaciation of the western Tienshan (Uzbekistan)]}

Abstract: Soil geographic studies were carried out in the Oigaing valley between Ugamsky and Pskemsky range NE of Tashkent (W-Tienshan, Republic of Uzbekistan) with special regard to the Pleistocene and Holocene glaciation. Clear end moraines of the last main glaciation are preserved at the junction of Maidan and Oigaing river at 1500-1600 $m$ a.s.l. They show intensively weathered soils with a depth

*) Anschrift der Verfasser: Prof. Dr. W. ZECH und Dr. R. BäUmLER, Lehrstuhl f. Bodenkunde u. Bodengeographie, Universität Bayreuth, D-95440 Bayreuth. Dr. O. SAvoskul, Geographisches Institut, Russische Akademie der Wissenschaften, Staromonetny per. 29, Moskau, 109017. Dr. A Ni und M. Petrov, Institut f. Geologie u. Geophysik, Akademie der Wissenschaften der Republik Usbekistan, ul. Mozozova 49, 700041 Taschkent. of more than $80 \mathrm{~cm}$. Similar (kp) sits of presumah)ly l'leistocene or late glacial origin are also located upvalley at the embouchure of numerous side valleys (Beschtor, Tekesch, Aütor) into the main valley of Oigaing. All side valleys are characterized by late glacial ground and end mo-raines in 2500-2700 $\mathrm{m}$ a.s.1. showing intensively weathered brown colored soils of $30-40 \mathrm{~cm}$ depth. Further moraines of Holocene or recent origin are located approach of the recent glaciers which descend to $3000-3200 \mathrm{~m}$. They show shallow, initial soils, and presumably correspond with glacial advances during the so-called "Little Ice Age" with a maximum advance at about 1850 in the Alps, and in the middle Holocene at about 2000 or 4000 a BP. Highly weathered, and rubefied interglacial soils developed from old Quaternary gravel are preserved above high glacial ice marginal grounds of the last main glaciation $(>2850 \mathrm{~m}$ a.s.1.) in the lower side valley of the Barkrak river. In the upper valley huge drift could be shown above the ice marginal grounds, but without typical forms of morainic deposits. They give evidence for older glaciations with a greater extent compared with the last main glaciation. However, no corresponding moraines are present in the working area.

\section{Einleitung}

Die aktuelle Diskussion über die Folgen der Zunahme klimarelevanter Spurengase geht einher mit verstärktem Interesse an der Erforschung zeitlich zurückliegender Klimaschwankungen. Methodisch gesehen gibt es hierfür verschiedene Ansätze, u. a. die Rekonstruktion früherer Gletscherschwankungen. Gletscher reagieren in der Regel besonders sensibel auf Veränderungen der Temperatur- und Niederschlagsverhältnisse. Vereinfacht gilt: Erwärmung führt zum Abschmelzen und damit zum Rückzug, Abkühlung hingegen korreliert mit Gletschervorstößen. Verständlicherweise liegen für das Holozän besonders viele Untersuchungen über Gletscherund Klimaschwankungen vor, z. B. aus den Alpen (Heuberger 1966, 1968, Zoller et al. 1966, Patzelt \& Bortenschlager 1978, Kerschner \& BerkTOld 1981), Skandinavien (ANDERSEN \& SOLLID 1971, KARLEN 1973, 1982, InNES 1984) oder Asien (HeubERGER 1956, RÖTHLISBERGER \& GEYH 1985, SHIRAIWA \& WATANABE 1991, Dyurgerov et al. 1994, KuHLe 1994).

Sie basieren in der Regel auf geomorphologischen Befunden, Radiocarbonanalysen, Pollenanalysen sowie lichenometrischen Erhebungen. Bodenkund- 
liche Untersuchungen zur Rekonstruktion früherer Gletschervorstöße sind vergleichsweise selten (BIRKELAND 1978, 1984, FitZe 1980, 1982, Mellor 1987, Bäumler et al. 1991, 1995).

Im folgenden berichten wir über bodengeographische Studien aus dem Westlichen Tienshan. Sie haben zum Ziel, die Ausdehnung glazialer Ablagerungen im Oigaing-, Tekesch- und Barkraktal zu erkunden. Spezielle bodenanalytische Ergebnisse werden zu einem späteren Zeitpunkt im Zusammenhang mit den pollenanalytischen, radiocarbonanalytischen und lichenometrischen Befunden vorgestellt.

\section{Ergebnisse}

\subsection{Das Oigaing- und Maidantal}

Abb. 1 informiert über die Lage des Untersuchungsgebietes, das etwa $150 \mathrm{~km}$ nordöstlich von Taschkent liegt, und zwar zwischen dem Ugamsky- und Pskemsky- und Alatai-Gebirge. Man erkennt, daß sich der Pskemfluß bei Saritschajak flußauf in den Maidan bzw. Oigaing aufspaltet. Knapp oberhalb des Zusammenflusses finden sich in 1600 bis $1650 \mathrm{~m}$ die ersten eindeutig als Stirnmoränen anzusprechen- den glazialen Ablagerungen (Nr. 1 und 2 in Abb. 1). Sie sitzen einer mit lößähnlichem Substrat bedeckten Schotterterrasse auf, die sich oberhalb der Einmündung des Maidan in den Oigaing erhalten konnte. In annähernd gleicher Höhenlage erkennt man die Stirnmoränen am Ausgang des Maidantales (Nr. 2 in Abb. 1). Sie sind sogar viel mächtiger als jene des Oigaingtales und bilden einen regelrechten Querriegel, den der Maidan kerbtalartig durchbricht. Unterhalb dieses Durchbruches findet sich bis auf etwa $1500 \mathrm{~m}$ herab ein geschiebereicher Schuttkegel. Im Gegensatz zu dieser Maidan-Moräne ist die OigaingStirnmoräne weniger imposant ausgebildet. Bei einer Gipfelflur von etwa $4000 \mathrm{~m}$ errechnet sich daraus nach der Methode von v. HöFer (1879) eine Schneegrenzdepression von 700-800 m für die jüngste Hauptvergletscherung. Gleiches gilt für die Anwendung der bei KuHLE (1994) beschriebenen Methode. Das entspricht der ELA(Schneegrenz-)Dcpression, die Grosswald et al. (1994) für die $400 \mathrm{~km}$ östlich anschließenden Massive des Tienshan und KuHLE (1994) für die $750 \mathrm{~km}$ südöstlich gelegenen Ketten von Karakorum und Kuenlun für das Spätglazial nachgewiesen haben. In Tab. 1 ist die Bodenbildung der Oigaing-Endmoräne in $1640 \mathrm{~m}$ be-

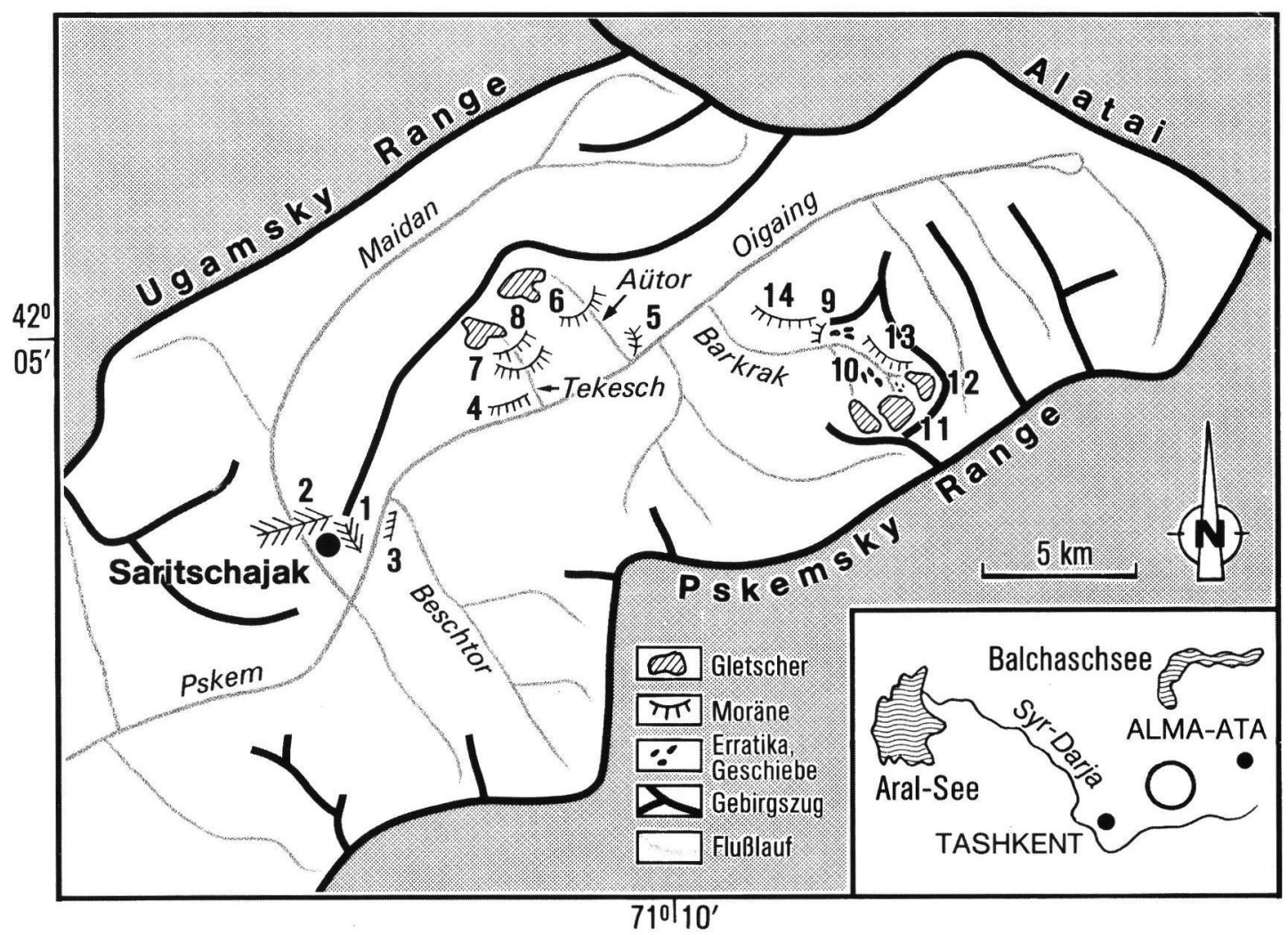

Abb. 1: Lage des Untersuchungsgebietes im westlichen Tien Shan, östlich von Tashkent (Republik Usbekistan; Erläuterung im Text).

Fig. 1: Location of the research area in the western Tienshan, east of Tashkent (Republic of Uzbekistan). 


\begin{tabular}{|c|c|c|c|c|c|c|c|c|c|c|c|c|c|}
\hline $\begin{array}{l}\text { Profil } \\
\text { Nr. }\end{array}$ & $\begin{array}{l}\text { Lage } \\
\text { (mutmaßi. stratigraphische Zuordnung) }\end{array}$ & $\begin{array}{l}\text { Höhe } \\
\text { mü.M. }\end{array}$ & $\begin{array}{l}\text { Ausgangs- } \\
\text { gestein }\end{array}$ & Horizonte & $\begin{array}{l}\text { Tiele } \\
\mathrm{cm}\end{array}$ & $\begin{array}{l}\text { Farbe } \\
\text { feldfrisch }\end{array}$ & Textur & Gefüge & Dichle & $\begin{array}{l}\text { Skelettgehalf } \\
\text { Vol\% }\end{array}$ & $\begin{array}{l}\mathrm{pH} \\
\%\end{array}$ & $\begin{array}{c}\text { Corg } \\
\%\end{array}$ & $\begin{array}{c}C_{\text {carb }} \\
\%\end{array}$ \\
\hline \multirow[t]{4}{*}{ TS 94/1 } & Wallmoräne (Nr. 9 in Abb. 1) & 2900 & Mischgestein: & Ah & 0.15 & grauschwarz & uls & krü-sin & Ld 1 & 50 & 6.8 & 4.31 & 1.17 \\
\hline & im rechten Talbodenbereich des Barkrak, & & Granit, wenig & B & $15 \cdot 40$ & hellbraun & uls & sub-sin & Ld 1 & 50 & 7.4 & 0.56 & 1.09 \\
\hline & (spätglazial) & & Carbonate & CvB & $40-60$ & graubraun & uls & $\sin$ & $\operatorname{Ld} 4$ & 75 & 7.5 & 0.43 & 1.38 \\
\hline & & & & $c$ & $60-90+$ & hellgrau & uls & $\sin$ & $\operatorname{Ld} 4$ & 75 & 7.7 & 0.35 & 1.02 \\
\hline \multirow[t]{4}{*}{ TS 94/2 } & Moränenablagerungen im Zwickel zwischen & 3260 & Mischgestein: & An & 0.5 & graubraun & s'u & $\mathrm{fkrü}$ & Ld 1 & 40 & 5.3 & 4.78 & 0.01 \\
\hline & mittlerem und rechtem Barkrakgletscher, & & Granit, Porphyr & B1 & $5-35$ & braun & IU & sub & $\operatorname{Ld} 3$ & 40 & 5.2 & 2.10 & 0.01 \\
\hline & von der Schuttzunge des neuzeitlichen & & & B2 & 35.55 & braun & IU & sub & $\operatorname{Ld} 3$ & 50 & 5.3 & 0.72 & 0.03 \\
\hline & Gletschers z.T. überfahren (mittelholozän) & & & $B C$ & $55 \cdot 70+$ & oliv & is & $\sin$ & $\operatorname{Ld} 5$ & 70 & 5.7 & 0.24 & 0.01 \\
\hline \multirow[t]{3}{*}{ TS $94 / 3$} & Moränenablagerungen ( $\mathrm{Nr} .10$ in Abb. 1) & 3100 & Mischgestein: & Ah & 0.6 & braunschwarz & sul & krü & $\operatorname{Ld} 3$ & 20 & 5.6 & 4.25 & 0.01 \\
\hline & des Barkrakgletschers links des Flusses & & Granit, Porphyr. & $A B$ & $6 \cdot 35$ & schwarzbraun & I's & $\sin -f$ krü & $\operatorname{Ld} 2$ & 40 & 4.9 & 1.23 & 0.01 \\
\hline & (mittelholozän) & & Gabbro & $B C V$ & $35-105+$ & graubraun & Is & sub & Ld 2-3 & 60 & 5.3 & 0.35 & $\ldots$ \\
\hline \multirow[t]{4}{*}{ TS $94 / 4$} & Eisrandleiste (Nr. 14 in Abb. 1) & 2870 & Mischgestein: & An & 0.7 & graubraun & sul & fkrü & $\operatorname{Ld} 2$ & 20 & 5.0 & 2.48 & 0.01 \\
\hline & im unteren Barkraktal, südwestexponierter Hang & & Granit, Kalk & B1 & $7 \cdot 27$ & braun & st & pol-sub & $\operatorname{Ld} 4$ & 25 & 5.4 & 0.70 & 0.01 \\
\hline & (hochglazial) & & & B2 & $27 \cdot 50$ & braun & st & sub & $\operatorname{Ld} 4$ & 30 & 5.5 & 0.49 & -- \\
\hline & & & & $\mathrm{Cr}$ & $50-87$ & grau & I's & $\sin$ & $L d 2$ & 50 & 6.5 & 0.28 & 0.04 \\
\hline \multirow[t]{5}{*}{ TS 94/5 } & Oberhalb der hochglazialen Ufermoräne & 2890 & Granifführende & An & 0.13 & graubraun & sul & f krü & $\operatorname{Ld} 2$ & 0 & 5.4 & 2.28 & 0.02 \\
\hline & (Nr. 14 in Abb. 1) mit Profil TS 94/4, & & Fließerde über & B1 & $13 \cdot 55$ & braun & sL & sub & $\operatorname{Ld} 3$ & $<10$ & 5.3 & 1.09 & 0.01 \\
\hline & unteres Barkraktal Interglazial, & & carbonatischen, & IIB1 & $55-85$ & rötl.braun & stL & sub & $\operatorname{Ld} 4$ & 10 & 5.1 & 0.51 & 0.01 \\
\hline & (Mitte//Spätpleistozän) & & älterquartären & II 82 & $85 \cdot 115$ & rotbraun & stL & sub & $\operatorname{Ld} 4$ & 20 & 5.3 & 0.37 & 0.01 \\
\hline & & & Konglomeraten & $\| \mathrm{Cr}$ & $115-135+$ & $\begin{array}{l}\text { gelblich- } \\
\text { braun }\end{array}$ & is & sub & $\operatorname{Ld} 5$ & 25 & 7.8 & 0.32 & 2.49 \\
\hline \multirow[t]{2}{*}{ TS 94/6 } & Stadiale Stirnmoräne (Nr. 8 in Abb. 1) & 2820 & Mischgestein: & An & 0.12 & schwarz & us & Krü & Ld 1 & 40 & 6.8 & 6.92 & 0.87 \\
\hline & $\begin{array}{l}\text { des Tekeschgletschers (holozän, } \\
\text { jünger als } 5000 \text { Jahre) }\end{array}$ & & $\begin{array}{l}\text { Granit, Gabbro } \\
\text { Porphyr, Kalk }\end{array}$ & $c_{v}$ & $12 \cdot 50+$ & grau & us & sin-sub & Ld1 & $60-70$ & 7.5 & 0.01 & 3.17 \\
\hline \multirow[t]{2}{*}{ TS 94/7 } & Stadiale Ufermoräne des & 2910 & Mischgestein: & $\mathrm{Ai}$ & 0.1 & dunkelgrau & $s$ & sub & Ld 1 & 80 & 7.5 & 0.23 & 0.29 \\
\hline & $\begin{array}{l}\text { Tekeschgletschers (neuzeitlich, evtl. } \\
1850 \text { ) }\end{array}$ & & $\begin{array}{l}\text { Granit, Gabbro, } \\
\text { Porphyr, Kalk }\end{array}$ & c & 1. $50+$ & grau & s & sub & $\operatorname{Ld} 4$ & 70 & 7.8 & 0.11 & 0.63 \\
\hline Pर्ता & $\frac{\text { lobe }}{\text { Lage }}$ & Höhe & $\begin{array}{l}\text { Popny, halik } \\
\text { Ausgangs- }\end{array}$ & Horizonte & Tiele & Farbe & Textur & Gefüge & Dichle & Skelettgehall & $\mathrm{pH}$ & Corg & $C_{\text {carb }}$ \\
\hline Nr. & (mutmaßl. stratigraphische Zuordnung) & m ü.NN & gestein & & $\mathrm{cm}$ & feldfrisch & & & & Vol\% & $\%$ & $\%$ & $\%$ \\
\hline \multirow[t]{3}{*}{ TS $94 / 8$} & Endmoräne ( $\mathrm{Nr} .7$ in Abb. 1) & 2680 & Mischgestein: & Ah & 0.15 & grauschwarz & ust & sub & $\operatorname{Ld} 2$ & 40 & 5.7 & 1075 & 0.02 \\
\hline & des Tekeschgletschers & & Granit, Gabbro, & B & $15-60$ & hellbraun & ust & sub & $\operatorname{Ld} 4$ & $60-70$ & 5.0 & 1.44 & 0.01 \\
\hline & (spätglazial) & & $\begin{array}{l}\text { Porphyr, carbonat- } \\
\text { haltig }\end{array}$ & $\mathrm{Cv}$ & $60-75+$ & grau & us & $\sin$ & -- & 80 & 7.6 & 0.18 & 0.62 \\
\hline \multirow[t]{5}{*}{ TS $94 / 9$} & Saritschajak, Stirnmoräne, (Nr. 1 in Abb. 1) & 1650 & Geschiebemergel & Ah & 0.40 & braunschwarz & ust & krü & $\operatorname{Ld} 2$ & $25-30$ & 7.4 & 399 & 0.98 \\
\hline & auf derSchotterterrasse oberhalb des & & mit Granit und & $A B$ & $40-60$ & braun & ust & sub & Ld 3 & 20 & 7.6 & 0.97 & 2.43 \\
\hline & Zusammenflusses von Oigaing und Maidan & & Porphyr & $\| B$ & $60-78$ & hellbraun & $u$ & sub & $\operatorname{Ld} 4$ & $<10$ & 7.6 & 0.79 & 2.07 \\
\hline & (hochglazial) & & carbonatreich & $\| \mathrm{BCc}$ & $78 \cdot 100$ & hellbraun & u & pol-sub & Ld 3-4 & $<10$ & 7.8 & 0.59 & 278 \\
\hline & & & über Schluff & $\| \mathrm{Crc}$ & $100-140+$ & $\begin{array}{l}\text { weißes Kalk- } \\
\text { mycel }\end{array}$ & $u$ & pol-sub & $\operatorname{Ld} 4$ & $<10$ & 7.8 & 0.37 & 4.30 \\
\hline \multirow[t]{3}{*}{ TS $94 / 10$} & Ufermoräne (Nr. 4 in Abb. 1) & 2160 & Mischgestein: & Ah & 0.20 & grauschwarz & ust & krü & Ld 2 & $20-30$ & -- & .- & .. \\
\hline & am S-exponierten Hang des Oigaingtales & & Granit, Gabbro & B & $20-64$ & braun & ust & sub & $\operatorname{Ld} 4$ & 60 & -- & -- & -- \\
\hline & unterhalb der Einmündung desTekesch (spätglazial) & & Porphyr, Kalk & $\mathrm{BCv}$ & $64 \cdot 70+$ & braungrau & us & $\sin$ & $\ldots$ & 80 & $\ldots$ & $\ldots$ & $\ldots$ \\
\hline \multirow[t]{4}{*}{ TS 94/11 } & Stirnmoränen (Nr. 5 in Abb. 1) & 2250 & blockreicher & Ah & 0.12 & grauschwarz & sul & krü & $\operatorname{Ld} 2$ & 25 & -- & .. & -. \\
\hline & im Mündungsbereich Aütor - Oigaing & & Geschiebemergel & Bv & $12 \cdot 40$ & braun & sul & sub & $\operatorname{Ld} 3$ & 45 & -- & -. & -. \\
\hline & (spätglazial) & & & $\mathrm{BCv}$ & $40-50$ & hellbraun & uls & sub & $\operatorname{Ld} 2$ & 60 & -- & -. & 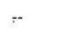 \\
\hline & & & & $\mathrm{Cr}_{\mathrm{v}}$ & $50.63+$ & hellgrau & is & $\sin$ & Ld 2 & 70 & .. & .. & ... \\
\hline
\end{tabular}

1) Ansprache: s. AG Bodenkunde, 1982 
schrieben (Profil TS 94/9). Man erkennt eine deutliche Zweischichtigkeit des Profils; ein skelettreicher A- und $\mathrm{AB}$-Horizont von $60 \mathrm{~cm}$ Mächtigkeit überdeckt schluffiges, carbonatreiches, skelettarmes Material, das im IIB-Horizont bis in $80 \mathrm{~cm}$ Bodentiefe verbraunt ist. Da wir unterhalb von Saritschajak keine vergleichbaren Moränenablagerungen fanden, ist davon auszugehen, daß die Maidan- und Oigainggletscher während der letzten Hauptvereisung bis auf etwa 1600 m herabreichen. Dafür spricht auch, daß die mächtige Schotterterrasse, welche sich im Zwickel zwischen Maidan- und Oigaingtal erhalten hat, von einer lößartigen, wahrscheinlich spätpleistozänen Schluffdecke überlagert ist, aus der sich schwarzerdeähnliche Böden entwickelt haben, die heute landwirtschaftlich genutzt werden. Dies bedeutet allerdings, daß die errechnete Schneegrenzdepression in den westlichen Randgebirgen des Tienshan um $400-500 \mathrm{~m}$ bzw. rund $40 \%$ unter den für Zentral- und Hochasien charakteristischen Werten für das letzte Hochglazial liegt (KuHLE 1994). Dafür gibt es mehrere Gründe. In der vorliegenden Studie wurden im Vergleich zu den Arbeiten in Zentralund Hochasien vergleichsweise kleine Einzugsgebiete mit einer deutlich niedrigeren Gipfelflur um etwa $4000 \mathrm{~m}$ ü. M. untersucht. Zum anderen sind die Niederschläge im westlichen Tienshan gering. Nach den Angaben der im Untersuchungsgebiet gelegenen zwei meteorologischen Stationen liegen-sie in der Nähe von Saritschajak bei 833,7 mm (1937-93; $1400 \mathrm{~m}$ ü. M.) und im Oigaing-Tal zwischen der Mündung des Aütor und Barkrak bei $779,5 \mathrm{~mm}$ (1989-93; 2200 m ü. M.).

Die talab im Pskemtal beschriebenen altquartären Moränen der sogenannten Nanaivergletscherung (VASILKOVSKIY 1951) konnten wir nicht verifizieren. Weitere glaziale Ablagerungen finden sich im Oigaingtal jedoch flußauf, aber nur vereinzelt, und zwar bevorzugt im Bereich der Einmündung S- oder N-exponierter Seitentäler. Dazu zählen z. B. die Ufermoränen in 1700 bis $1720 \mathrm{~m}$ Höhe bei Beschtor unterhalb der Einmündung des Beschtorbaches (Nr. 3 in Abb. 1) in das Oigaingtal sowie geschiebereiche, wallförmige Hangverflachungen in $2160 \mathrm{~m}$ am südexponierten Hang unterhalb des Zusammenflusses von Tekesch und Oigaing (Nr. 4 in Abb. 1). Letztere weisen Braunerden auf mit einer bis zu $60 \mathrm{~cm}$ in die Tiefe reichenden Verbraunung (Tab. 1, Profil TS 94/10). Vermutlich handelt es sich um Ufermoränen des Haupttalgletschers. Sie liegen $60-80$ m über dem rezenten Oigaingflußbett.

Sehr gut sind die Moränenwälle am Ausgang des Aütortales in 2200 bis $2280 \mathrm{~m}$ erhalten (Nr. 5 in Abb. 1). Es handelt sich aber nicht um Ufermoränen des Oigainghaupttalgletschers, sondern um Stirnmoränen des Aütorgletschers. Dafür sprechen 1. die bogenförmig in das Aütortal orographisch links hinein- ziehenden Wälle, sowie 2. die Tatsache, daß die Moränen keine granitischen Gesteine aufweisen, sondern überwiegend Carbonate. Im Oigainghaupttal dominieren dagegen vielfach rote Granite, die sich nur im Sockelbereich der Aütormoränen nachweisen lassen. Aufgrund der Bodenbildung (die Horizontfolge lautet $\mathrm{Ah}[12 \mathrm{~cm}], \mathrm{Bv}[28 \mathrm{~cm}], \mathrm{BCv}[10$ cm], Cv [13 cm+], Tab. 1, Profil TS 94/11) dürfte es sich um Ablagerungen eines spätglazialen Vorstoßes des Aütorgletschers handeln, der mit einer Schneegrenzdepression von etwa $400 \mathrm{~m}$ einherging. Eine entsprechende Schneegrenzabsenkung liegt zwischen den Werten, die nach Kunle (1994) als charakteristisch für das frühe Spätglazial (17000$13000 / 10000$ a BP; 700-1100 m) und für holozäne Gletschervorstöße zwischen 5500 b is 1700 a BP $(80-300 \mathrm{~m})$ in Hoch- und Zentralasien angegeben werden. Auch hier beträgt der Unterschied mindestens $300 \mathrm{~m}$ bzw. rund $40 \%$.

Eine weitere spätglaziale bis frühholozäne, gut bewachsene Moräne findet sich im Aütortal oberhalb der soeben beschriebenen Moränen in $2500 \mathrm{~m}$, worauf wir bei der Besprechung des Tekeschtals nochmals zurückkommen werden (Nr. 6 in Abb. 1). Die wallförmigen Stirnmoränen an der Einmündung des Aütor in den Oigaing werden von Terrassenschottern des Haupttales „umflossen“. Diese Schotter enden etwa $30 \mathrm{~m}$ über der rezenten Talsohle. Hierbei handelt es sich um jüngere Aufschüttungen, da die Schotter nur ein Ah-C-Bodenprofil aufweisen, eine Verbraunung somit fehlt. Ihre Genese könnte mit einer jüngeren Haupttalverschüttung infolge eines Bergsturzes oberhalb der Tekescheinmündung im Zusammenhang stehen.

Neben dem Oigainghaupttal untersuchten wir genauer das südexponierte Tekesch- und das nordexponierte Barkraktal. Zunächst schildern wir die Ergebnisse aus dem Tekeschtal.

\subsection{Das Tekeschtal}

Der Tekesch mündet in $2100 \mathrm{~m}$ in den Oigaing (Abb. 1). Wie bereits erwähnt, befinden sich unterhalb der Einmündung auf dem rechten, südexponierten Hang in 2160 bis $2180 \mathrm{~m}$ blockreiche Hangverflachungen, die wir als spätglaziale Ufermoränen des Oigainggletschers interpretieren. Im Tekeschtal selbst fällt eine weitere mächtige wallförmige, gut bewachsene, geschiebereiche Endmoräne in 2500 bis $2600 \mathrm{~m}$ ins Auge. Auch sie weist etwa 50 bis 60 $\mathrm{cm}$ mächtige, blockreiche Braunerden auf, deren Genese wohl ebenfalls mindestens bis ins Frühholozän, vermutlich sogar bis in das Spätglazial zurückreicht (Tab. 1, Profil TS 94/8). Diese Moräne, die sich bereits außerhalb der Datierbarkeit mittels Lichenometrie befindet (d. h. älter als 4000 a BP; SAvoskUL 1996), liegt somit in der gleichen Höhenlage 
wie jene im Aütortal. Der entsprechende Gletschervorstoß ist mit einer Schneegrenzdepression von rund $250 \mathrm{~m}$ einhergegangen. Oberhalb der spätglazialen bis frühholozänen Tekeschmoränen in 2600 $\mathrm{m}$ lassen sich drei weitere Stadien identifizieren, und zwar in $2820 \mathrm{~m}$ (Nr. 8 in Abb. 1; grasbewachsen, Horizontfolge Ah- $\mathrm{C}$, ohne Verbraunung, Tab. 1, Profil TS 94/6), in $2910 \mathrm{~m}$ (vegetationsfrei, mit mächfigen, scharfen Konturen, Profilfolge Ai-C, Tab. 1, Profil TS 94/7), sowie in $2930 \mathrm{~m}$ (schwach ausgebildet, nicht bewachsen, Horizontfolge Ai-C), in relativer Nähe zur rezenten Gletscherzunge bei $3000 \mathrm{~m}$. Aufgrund der fehlenden Verbraunung des Solums der letztgenannten drei Moränenstadien und unter Berücksichtigung der morphologischen Gegebenheiten interpretieren wir diesen Befund wie folgt: (vgl. dazu auch SAVOSKul \& DreChSEL 1994).

2930 m neuzeitlich, erinnern dem Aussehen nach an die 1920er Moränen in den Alpen (ELADepression $35 \mathrm{~m}$ )

$2910 \mathrm{~m}$ neuzeitlich, erinnern dem Aussehen nach an die 1850er Moränen in den Alpen (ELADepression $45 \mathrm{~m}$ )

2820 m holozän (evtl. 2000 a BP; ELA-Depression $90 \mathrm{~m}$ )

2500 bis $2600 \mathrm{~m}$ spätglazial bis frühholozän (ELADepression $250 \mathrm{~m}$ )

Betrachten wir im folgenden die Befunde aus dem nordexponierten Barkrakseitental.

\subsection{Das Barkraktal}

Der Barkrak mündet von Südosten kommend bei $2200 \mathrm{~m}$ in den Oigaing (Abb. 1). Erste zweifelsfreie Moränen (Nr. 9 in Abb. 1) beginnen im Talbodenbereich etwa bei $2700 \mathrm{~m}$. Ihrer Gestalt nach erinnern sie an Grundmoränen mit Buckel/Muldenrelief, jedoch treten vereinzelt auch Wallformen in Erscheinung. Man hat durchaus den Eindruck, daß ein mehrphasiger Gletschervorstoß für diese Moränen verantwortlich ist. Sie enden talaufwärts in etwa 2900 m. Durch Hangschutt und Bergsturzmaterial aus den westexponierten Wänden wurde dieser Moränengürtel $z$. T. wieder zerstört bzw. überdeckt. Nach Tab. 1 (Profil TS 94/1) haben sich aus diesen überwiegend granitischen und schwach carbonathaltigen Gesteinen blockreiche Braunerden entwickelt mit einer Verbraunungstiefe bis zu $60 \mathrm{~cm}$. Dies spricht für ein spätglaziales bis frühholozänes Alter der Moränen. Die errechnete Schneegrenzdepression beträgt ca. $275 \mathrm{~m}$, was mit jener der Moränen Nr. 6 im Aütortal bzw. mit jener der Moränen Nr. 7 im Tekeschtal korreliert (Abb. 1).

Weiter taleinwärts treten in $3100 \mathrm{~m}$ Höhe auf der linken, und etwas höher auch auf der rechten
Bachseite, wiederum Blockgletschermoränen mit Buckel/Muldenrelief in Erscheinung (Nr. 10 in Abb. 1). Auch die Böden dieser Moränen sind kräftig verbraunt (Horizontfolge Ah $0-6 \mathrm{~cm}, \mathrm{AB} 6-35 \mathrm{~cm}, \mathrm{BCV}$ $35-105 \mathrm{~cm}$, s. Tab. 1, Profil TS 94/3). Von der Bodenentwicklung her gesehen, könnten diese Böden spätglazialer Genese sein. Ähnliches gilt für die Böden im Zwickelbereich zwischen dem mittleren und rechten Barkrakgletscher in $3260 \mathrm{~m}$ Höhe (Tab. 1, Profil TS 94/2), die bis zu $55 \mathrm{~cm}$ Bodentiefe verbraunt sein können. Zieht man jedoch das gesteinsbedingt bereits lehmig-tonige, teilweise schon verbraunte Substrat der C-Horizonte der neuzeitlichen Moränen im Barkrak-Tal mit in die Betrachtung ein, so ist ein mittelholozänes Alter der bis 3100 m herabziehenden Moränen nicht auszuschließen. Unterstützt wird diese Vorstellung durch die Radiocarbondatierung eines fossilen Ah-Horizontes, der in 70 - $80 \mathrm{~cm}$ Bodentiefe vor, d. h. talabwärts des nachfolgend beschriebenen neuzeitlichen Blockgletschers beprobt wurde. Die Analyse ergab ein ${ }^{14} \mathrm{C}-\mathrm{Al}$ ter der Huminsäurefraktion von $2850 \pm 110$ Jahren $\mathrm{BP}$

Nur etwa $150 \mathrm{~m}$ höher liegt südöstlich bei $3250 \mathrm{~m}$ eine morphologisch sehr frische, nicht bewachsene, riesige Schuttzunge, die zum mittleren Barkrakgletscher gehört (Nr. 11 in Abb. 1). Frische, an die 1850er-Moränen der Alpen erinnernde Wallkonturen fehlen weitgehend. An der Front, d. h. am untersten Ende bricht die Schuttzunge mit steilen, hellen Halden ab, und Wasser quillt aus dem Schutt. Ohne Zweifel liegt hier eine schuttbedeckte Gletscherzunge vor, die alle Merkmale eines noch nicht konsolidierten Blockgletschers aufweist. Trotz kräftiger Verwitterung des aufliegenden Schutts spricht das Fehleneiner Bodenvegetation für ein neuzeitliches Alter. Im Gegensatz zum mittleren Barkrakgletscher zeigt der östlich davon gelegene „rechte“ Barkrakgletscher (Nr. 12 in Abb. 1) markante und frische, scharfe Formen, die durchaus an die 1850er Moränen in den Alpen erinnern. Die Bodenbildung ist über das Syrosemstadium nicht hinausgekommen, auch fehlt eine Bodenvegetation. Zwischen "mittlerem" und „rechtem" Barkrakgletscher finden sich noch kleinere, z. T. mit Bergsturzmaterial bedeckte Schuttzungen.

Zusammenfassend gilt, daß im Talbodenbereich das Barkrak drei Gletscherstände rekonstruiert werden können:

$3250 \mathrm{~m}$ Beginn der Schutt- und Eiszunge des neu zeitlichen mittleren Barkrakgletschers $(\mathrm{Nr}$. 11 in Abb. 1)

3100 m mittelholozäne Moränen des mittleren Bar krakgletschers (Nr. 10 in Abb. 1)

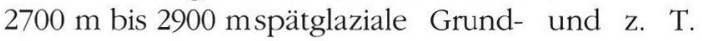
auch Stirnmoränen des mittleren Barkrakgletschers (Nr. 9 in Abb. 1) 
Neben Grund- und Stirnmoränen kommen im Barkraktal auch Reste von Ufermoränen vor, und zwar am rechten, süd- bis südwest-exponierten Hang ( $\mathrm{Nr}$. 13 in Abb. 1). Von der Schutt- und Eiszunge in 3250 m Höhe des mittleren Barkrakgletschers (Nr. 11 in Abb. 1) folgt nach Norden zunächst das Buckel/Muldenrelief der vermutlich spätglazialen Grundmoräne, die kräftiges Graswachstum und bis zu $50-60 \mathrm{~cm}$ verbraunte Böden aufweist. Hangaufwärts folgen drei stufenartige Verflachungen, und zwar in etwa $3250 \mathrm{~m}, 3350 \mathrm{~m}$ und $3400 \mathrm{~m}$. Diese Verflachungen ziehen mit geringem Gefälle talabwärts, d. h. nach Nordwesten. Sie weisen reichlich Geschiebe auf, hangaufwärts folgen dagegen steinarme, kräftig vergraste Bänder. Diese Befunde deuten wir als Eisrandzeugen spätglazialen oder hochglazialen $\mathrm{Ur}$ sprungs. Der obere Eisrandrest bei $3400 \mathrm{~m}$ befindet sich etwa $300 \mathrm{~m}$ über dem rezenten Barkrakbach.

Bergauf ist der Hang bis in Höhen von $3800 \mathrm{~m}$ mit Geschiebe übersät, ohne daß wallförmige Ablagerungen nachzuweisen sind. Erst oberhalb von 3800 m werden die Geschiebe von eckig-kantigem Frostschutt abgelöst. Dieser Befund spricht möglicherweise für eine mächtige, vermutlich mittelpleistozäne Vergletscherung, die wesentlich größere Dimensionen angenommen hat als die spätpleistozäne.

Wie erwähnt, lassen sich diese Eisrandleisten, z. T. mit Unterbrechungen, gut talauswärts verfolgen. In 2870 m weist die oberste Verflachung (Nr. 14 in Abb. 1) eine bis $40 \mathrm{~cm}$ Tiefe reichende Verbraunung auf (Tab. 1, Profil TS 94/4). Oberhalb dieser Leisten sind die Böden jedoch bis über $1 \mathrm{~m}$ verbraunt und mit bis zu $30 \%$ Ton wesentlich tonreicher (Tab. 1, Profil TS 94/5). Auch im Vergleich zu Profil TS 94/9 bei Saritschajak in $1650 \mathrm{~m}$ ü. M. ist Profil 5, obwohl etwa $1200 \mathrm{~m}$ höher gelegen, wesentlich intensiver verwittert. Dies ist ein Hinweis darauf, daß die oberste Hangverflachung den Hochstand der letzten Hauptvergletscherung dokumentiert. Das tiefbraune, mächtige Solum außerhalb bzw. oberhalb der höchsten Eisrandleiste ist auf die intensive Bodenbildung während eines Interglazials zurückzuführen. Das Profil ist zweischichtig aufgebaut: die oberen $55 \mathrm{~cm}$ interpretieren wir als spätpleistozäne Solifluktionsdecke, welche die Reste eines geköpften, aus älterquartären Konglomeraten entstandenen in situBodenprofils überdeckt.

Oberhalb der vermutlich würmzeitlichen Ufermoränen in 2870 m treten im Kammbereich um 2900 $3000 \mathrm{~m}$ diese vermutlich älterquartären Konglomerate an die Oberfläche. Häufig sind sie mit Geschiebe bedeckt, ohne daß sich jedoch leistenförmige Hangverflachungen erkennen lassen, was wir bereits aus dem oberen Barkraktal beschrieben haben. Geschiebe dieser Art oberhalb der höchsten erkennbaren Eisrandspuren führen wir auf ältere Vereisun- gen zurück. Eindeutige Kritzung konnten wir nicht identifizieren, da die Gesteinsoberflächen stark angewittert sind.

Aus glazialmorphologischer Sicht könnte es sich aber auch um Eisrandleisten eines spätglazialen Stadiums handeln. Dafür sprechen neben einer geschätzten Eismächtigkeit von lediglich $300 \mathrm{~m}$ zwischen den obersten Eisrändern und dem rezenten Bachbett die berechneten Werte für die ELA-Absenkung und auch die zahlreichen Erratika und Geschiebeblöcke oberhalb der höchsten Eisrandlagen bis $3800 \mathrm{~m}$, allerdings ohne markierten Eisrand mit Gelände- oder Moränenkante. Dies steht aber im Widerspruch zu den bodenkundlichen Befunden, die bei einer Berücksichtigung der Höhendifferenz von knapp $1300 \mathrm{~m}$ zwischen Profil 9 bei Saritschajak und Profil 5 im Barkrak-Tal oberhalb der höchsten erkennbaren Eisrandspuren unter der Annahme gleichen Alters beider Ablagerungen ab- und nicht zunehmende Verwitterungsintensität bei steigender Meereshöhe aufweisen müßten, wie dies an der Südabdachung der zentralasiatischen Gebirgsmasse eindeutig nachgewiesen wurde (BäUMLER et al. 1991 und 1996, Bäumler 1993). Die Unterschiede in der Bodenentwicklung zwischen Profil 4 aus den Ablagerungen des höchstgelegenen Eisrandes am Unterlauf des Barkrak in $2870 \mathrm{~m}$ ü. M. und Profil 9 in 1650 $m$ ü. M. sind dagegen - ähnliches Alter der Ablagerungen vorausgesetzt - gut mit den unterschiedlichen Verwitterungsbedingungen in Abhängigkeit von der Meereshöhe und aufgrund von der Distanz der Ablagerungen vom Einzugsgebiet der Gletscher zu erklären. Profil 9 ist im Vergleich zu Profil 4 etwas stärker verwittert und das mitgeführte Geschiebe ist größtenteils aufgemahlen, was sich in einem geringeren Sand- und höheren Mittel- und Feinschluffanteil, nicht jedoch in höheren Tongehalten manifestiert. Die glazialgeomorphologischen Befunde deuten wiederum darauf hin, daß es sich bei Saritschajak um Ablagerungen der letzten Hauptvergletscherung handelt, obwohl die berechnete Schneegrenzdepression in bezug auf die in Hoch- und Zentralasien gefundenen Werte für ein spätglaziales Stadium spricht. Für eine eindeutige Klärung dieser Antimonie zwischen den glazialgeomorphologischen und bodenkundlichen Befunden sind aus unserer Sicht weitergehende Untersuchungen erforderlich.

\section{Schlußfolgerungen}

Überblickt man diese Ergebnisse, so ist festzuhalten, daß bodengeographische Untersuchungen einen wichtigen Beitrag leisten können zur Identifizierung glazialer Ablagerungen. Sie haben deshalb für die Rekonstruktion von Gletscher- und Klimaschwankungen eine große Bedeutung. Untersuchungen dieser Art sollten begleitet werden von quantitativen 
Aussagen zur Verwitterungsintensität, sowie lichenometrischen und pollenanalytischen Erhebungen. Bodengeographische Befunde können jedoch absolute Altersdatierungen z. B. mittels Radiocarbonanalysen nicht ersetzen.

Im Vergleich zu den bisherigen Vorstellungen über das Ausmaß der Vergletscherung im Tienshan gilt, daß die Untergrenze der jüngsten Hauptvergletscherung im engeren Arbeitsgebiet nicht bei 2000 bis $2500 \mathrm{~m}$ liegt (ZaBirov 1958, KorZHENEvSKIy 1960, Popov 1960, Grigorenko 1970), sondern bei 1500 bis $1600 \mathrm{~m}$, was gut mit Befunden von Heuberger (persönliche Mitteilung) im nördlichen Tienshan (Kirgisien, südl. von Bischkek) und von Grosswald et al. (1994) in der Region um den Issyk-Kul See übereinstimmt. Die morphologischen Gegebenheiten im Barkraktal deuten jedoch darauf hin, daß frühere Vergletscherungen wesentlich intensiver waren als jene der letzten Hauptvergletscherung (vgl. auch Popov 1960, GRIGoRENKo 1970).

\section{Zusammenfassung}

Die Gletscher im Oigaing- und Maidantal im Westlichen Tienshan (Usbekistan) reichten während der letzten Hauptvergletscherung bis 1500/1600 m herab, wie Endmoränen eindeutig belegen. Die Böden dieser Moränen sind bis etwa $80 \mathrm{~cm}$ Tiefe verbraunt. Im Oigainghaupttal finden sich weitere geschiebereiche Ablagerungen, so z. B. bei Beschtor in 1700 $1720 \mathrm{~m}$ (Nr. 3 in Abb. 1) und an der Einmündung des Tekesch $(2160 \mathrm{~m})$ bzw. Aütors $(2200-2280 \mathrm{~m})$ in den Oigaing (Nr. 4 bzw. 5 in Abb. 1). Sie sind vermutlich hochglazialer bzw. spätglazialer Genese

Die Seitentäler des Tekesch, Aütor und Barkrak weisen in 2500 bis $2700 \mathrm{~m}$ jeweils spätglaziale Stirnbzw. Grundmoränen auf (Nr. 7, 6, 9 in Abb. 1), im Tekesch- und im Barkraktal zusätzlich in $2810 \mathrm{~m}$ (Nr. 8 in Abb. 1) bzw. 3100 m Höhe (Nr. 10 in Abb. 1) mittelholozäne Ablagerungen.

Oberhalb (> $2850 \mathrm{~m}$ ) der hochglazialen Eisrandleisten (Nr. 14 in Abb. 1) finden sich im unteren Barkraktal interglaziale Böden aus älterquartären Schottern. Der Profilaufbau läßt Schichtigkeit erkennen mit einer verbraunten Solifluktionsdecke über einem in situ Bv-Horizont. Die Verbraunung reicht bis in $105 \mathrm{~cm}$ Bodentiefe.

Die Eismächtigkeit lag im Barkrak-Tal überschlagsweise bei $300 \mathrm{~m}$, bezogen auf die Höhendifferenz zwischen der obersten identifizierbaren Eisrandleiste und der rezenten Talsohle.

Oberhalb der Eisrandleisten (Nr. 13 in Abb. 1) in $3400 \mathrm{~m}$ im oberen Barkraktal finden sich bis $3800 \mathrm{~m}$ zwar keine wallförmigen Moränen oder Geländebzw. Moränenkanten, jedoch reichlich z. T. sehr mächtige Erratika und Geschiebe. Sie dokumentieren ältere Vergletscherungen, die wesentlich mäch- tiger waren als jene der jüngsten Hauptvergletscherung. Die korrespondierenden Moränen konnten wir nicht identifizieren.

\section{Dank}

Unser verbindlichster Dank gilt der Deutschen Forschungsgemeinschaft für die finanzielle Förderung dieser Untersuchung (436 RUS 113/6/1). Den Mitgliedern der Akademie der Wissenschaften in Tashkent, Abteilung Glaziologie, danken wir für die großzügige und freundschaftliche Unterstützung während der Geländearbeiten.

\section{Schriftenverzeichnis}

AG Bodenkunde (1982): Bodenkundliche Kartieranleitung (3. Aufl.). Hannover, $331 \mathrm{~S}$.

ANDERSEN, J. L. \& SOLLID, J. L. (1971): Glacial chronology and glacial geomorphology in the marginal zones of the glaciers, Midtdalsbreen and Nigardsbreen, South Norway. Norsk geogr. Tidsskr. 25: 1-38.

Bäumler, R. (1993): Bodenbildung und Verwitterungsintensität auf Moränen und Gletscherablagerungen im Khumbu Himal und oberen Solu-Tal, Ostnepal, Innsbrucker Geograph. Studien 20: 29-45.

Bäumler, R., Zech, W., Heuberger, H. \& Weber-Diefenbach, K. (1991): Investigations on the intensity of weathering of soils developed from glacial and fluvioglacial deposits and their relationship with the history of the landscape in the Mt. Everest region. Geoderma 48: 223-243.

Bäumler, R., Kemp-Oberhettinger, M., ZECH, W., Heuberger, H., Siebert, A., Madhikarmi, D. P. \& Poudel, K. P. (1996): Soil weathering on glacial and glaciofluvial deposits in the Langtang valley (Central Nepal) and its relation to the glacial history. Z. Geomorphologe N. F. 103: 373-387.

Birkeland, P. W. (1978): Soil development as an indication of relative age of quaternary deposits, Baffin Island, N.W.T., Canada. Arctic and Alpine Research 10: 733747.

- (1984): Holocene soil chronofunctions, Southern Alps, New Zealand. Geoderma 34: 115-134.

Dyurgerov, M. B., Mikhalenko, V. N., Kunakhovitch, M. G., Ushnurtsev, S. N., Liu, C. \& Xie, Z. (1994): On the Cause of Glacier Mass Balance Variations in the Tian Shan Mountains. GeoJournal 33.2/3: 311-317.

FITZE, P. (1980): Zur Bodenentwicklung auf Moränen in den Alpen. Geographica Helvetica 3: 97-106.

- (1982): Zur Relativdatierung von Moränen aus der Sicht der Bodenentwicklung in den kristallinen Zentralalpen. Catena 8: 265-306.

Grigorenko, P. G. (1970): The main features of the geological history of Kirgizskiy Tian-Shan in the Pleistocene. Materials on the geology of Caenozoic and recent tectonics of Tian-Shan, Frunze, 5-23 (in Russian).

Grosswald, M. G., Kuhle, M. \& Fastook, J. L. (1994): Würm glaciation of lake Issyk-Kul area, Tian Shan Mts.: A case study in glacial history of Central Asia. GeoJournal 33: $273-310$.

Heuberger, H. (1956): Beobachtungen über die heutige und eiszeitliche Vergletscherung in Ost-Nepal. Zeitschrift für Gletscherkunde und Glazialgeologie 7: 175185 . 
Heuberger, H. (1966): Gletschergeschichtliche Untersuchungen in den Zentralalpen zwischen Sellrain und Ötztal. Innsbruck und München: Wissenschaftliche Alpenvereinshefte 20: 126.

- (1968): Die Alpengletscher im Spät- und Postglazial. Eiszeitalter und Gegenwart 19: 270-275.

HÖFER, H. v. (1879): Gletscher und Eiszeitstudien. Sitzungsbericht d. Akademie d. Wissenschaften Wien, math.phys. Klasse I 79: 331-367.

INNES, J. L. (1984): Relative Dating of Neoglacial Moraine Ridges in North Norway. Zeitschrift für Gletscherkunde und Glazialgeologie 20: 53-63.

KARlen, W. (1973): Holocene glacier and climatic variations, Kebnekaise Mountains, Swedish Lappland. Geogr. Annaler 55A: 29-63.

- (1982): Holocene glacier fluctuations in Scandinavia. Striae 18: 26-34.

Korzhenevskiy, N. L. (1960): The nature of Central Asia. Tashkent, 22-30 (in Russian).

Kerschner, H. \& Berktold, E. (1981): Spätglaziale Gletscherstände und Schuttformen im Senderstal, Nördliche Stubaier Alpen, Tirol. Zeitschrift für Gletscherkunde und Glazialgeologie 17: 125-134.

KuHle, M. (1994): Present and Pleistocene Glaciation on the North-Western Margin of Tibet between the Karakorum Main Ridge and the Tarim Basin, Supporting the Evidence of a Pleistocene Inland Glaciation in Tibet. GeoJournal 33.2/3: 133-272.

Mellor, A. (1987): A pedogenic investigation of some soil chronosequences on neoglacial moraine ridges, Southern Norway: Examination of soil chemical data using principal component analysis. Catena 14: 369-381.

Patzelt, G. \& Bortenschlager, S. (1978): Zur Chronologie des Spät- und Postglazials im Ötztal und Inntal (Ostal- pen, Tirol). In: B. Frenzel (Hrsg.). Führer zur Exkursionstagung des IGCP-Projekts 73/1/24 „Quaternary Glaciations in the Northern Hemisphere“, 5. - 13. Sept. 1976, Bonn-Bad Godesberg, 185-197.

Popov, V. V. (1960): Stratigraphy of Antropogen in Tian Shan. Reports of the Institute of Geology, Academy of Sciences USSR 26: 116-126 (in Russian).

RÖTHL.IsBerger, F. \& GEYH, M. A. (1985): Glacier Variations in Himalayas and Karakorum. Zeitschrift für Gletscherkunde und Glazialgeologie 21: 237-249.

Savoskul, O. S. \& Drechsel, P. (1994): To the history of glaciation of the Pskem river basin in the Holocene. Materials of Glaciological Studies 78: 70-78.

- (1996): Lichenometric evidence of the Late Holocene glacier variations in the Oigaing river basin, Western Tian Shan, Central Asia. Zeitschrift f. Gletscherkunde und Glazialgeologie, eingereicht.

Shiraiwa, T. \& Watanabe, T. (1991): Late Quaternary Glacial Fluctuations in the Langtang Valley, Nepal Himalaya. Reconstructed by relative Dating Methods. Arctic and Alpine Research 23: 404-416.

VAsILKOVSKIY, N. P. (1951): To the question of age division of Quaternary deposits of NE Uzbekistan. Report of the Institute of Geology, Uzbekish Academy of Sciences, Tashkent, 5-44 (in Russian).

ZABIROv, R. D. (1958): Glaciation of Central Asia. Reports of Tian-Shan physical geographical station, Frunze, 56-72 (in Russian).

Zoller, H. Schindler, C. \& Röthlisberger, H. (1966): Postglaziale Gletscherstände und Klimaschwankungen im Gotthardmassiv und Vorderrheingebiet. Verh. d. Naturf. Ges. Basel 77: 97-164.

Manuskript eingegangen am 19.06. 1995 